\title{
THE UICC HOME TELEPHONE ACCESS SYSTEM
}

by Joseph F. Morrison

The purpose of this article is not to announce some startling new discovery, but to relate the experience at the University of Illinois Circle Campus with home telephone access to language lab recordings. The UICC system, started January 1967, has been experimental in nature, and the findings and conclusions of the experiment are summarized herein. The Audio Information Service (formerly Language Laboratory) is so called because, as in many other institutions, it provides audio materials for many disciplines - not only foreign languages.

\section{Rationale}

The extension of the language laboratory to the student's home is by no means a new idea. Still, it was not utilized at UICC, until increasing enrollments and additional course materials necessitated expansion of the Audio Information Service (AIS), whose basic system (Chester Electronic Labs) consists of three labs. There are two sixty-student-station audio active labs with dial access to 30 tape playback machines and one 15-student-station audio active record lab (non-dial access, Robert C. Merchant Electronics). The physical arrangement of the labs is such that addition of more student stations is impossible in the same building (Grant Hall). Thus, development of the UICC telephone access system has been motivated basically by need for expanded service and lack of funds to implement such expansion by adding student stations.

\section{Version I}

The possibility of students calling from their home telephones for language practice tapes was considered. Preliminary conferences held with Illinois Bell Telephone Company technicians led to the conclusion that the Western Electric KS-19522 recording coupler would lend itself to the proposed application. In as much as the KS-19522 can accommodate only one caller at a time, the problem then became one of development of a means to connect a tape playback machine to the recording coupler so that the following functions could be performed: 1) The playback machine automatically starts when called; 2) the machine automatically rewinds, stops, and prepares itself for the next call when the current call terminates; 3 ) the 
caller receives no answer if the system is inoperative. The KS-19522 provided all necessary control signals and the Chester CTM641 tape transport was connected to the coupler by means of an interface network developed by the AIS.

The system went into operation in January, 1967 with three separate couplers connected to three tape transports. A group of approximately ninety students in three different courses were asked to use the system during the quarter, and, when surveyed, this experimental group indicated great enthusiasm for the project. From the student point of view convenience was cited as the main advantage, since the current lesson was available 24 hours per day, 7 days per week. If the student lived within the Chicago city limits there was no toll charge involved for the call.

Disadvantages of the first system included: 1) Only one caller could obtain a practice lesson at a time; 2 ) additional callers received a busy signal if the line was in use; 3 ) it was necessary to have a separate tape machine for each lesson transmitted over a telephone line. Since the same lesson was available to the on-campus facility as well as the students at home, two machines were required to play the same lesson, one for each medium. Expansion of the home telephone service on that basis would have been very costly.

\section{Version II}

During the following year the system presently in use was designed. Illinois Bell provided a special "group listening arrangement," a circuit similar to that used in the recorded weather reporting service. The group listening arrangement involves a common bridging network in the local central office and can accommodate from 5 to 30 simultaneous incoming calls per tape. The rental cost of the equipment is determined by the number of terminals for incoming calls. In most cases, 5 terminals are adequate for a group of approximately 300 students who are required to call four times per week. In general, the number of terminals required is based on: 1) number of users; 2) number of expected calls per unit of time; 3) length of the lesson involved. During the same year a special switching interface was designed and manufactured at UICC, thus eliminating the duplication of tape machines previously mentioned. With the special interface, the same tape machine now serves both the on-campus facility and the telephone network. Further, the switching interface can connect any one of the 30 tape transports to any of the telephone lines in crossbar fashion. This feature allows quick restoration of service in the event of tape machine failure. The single access system (Version I) utilizing the KS-19522 coupler is still used for small groups or for special individual requests. The switching interface has a total capa- 
city of 15 group listening arrangements and 5 single access lines. If 30 terminals per group listening arrangements were connected in the central office, 455 callers could listen to 20 different tapes simultaneously.

\section{Applications}

The group listening arrangement is intended primarily to accom. modate large groups of students in first and second-year language courses. However, approximately 2000 Elementary Biology students also use the service to obtain their weekly lectures. In addition, the UICC Communicator (312-996-5500), a daily recorded announcement of events of general interest to the university community, uses the group listening arrangement. Recorded materials associated with many courses (including French, German, Italian, Polish, Russian, Spanish, Swahili, Biology, Vocabulary Improvement) and the UICC Communicator are available by home telephone.

\section{Problems Encountered}

Although the group listening arrangement and the new switching interface eliminate many of the original disadvantages, improvements are necessary. The main problem with the group listening arrangement is that the first caller will start the tape machine and any subsequent callers will hear the lesson in progress. This is not an important consideration for short lessons or messages such as the Communicator or language pattern drills. However, materials requiring sequential continuity present a special problem with respect to the group listening arrangement. This is overcome by resetting the tape at a regular interval and informing all users when the material will be available from the start. The resetting process is fully automatic, and, during the tape reset time all calls are switched to a special announcement tape. The audio fidelity of the telephone access arrangement is less than standard language lab fidelity. Signal bandwidth applied to the caller's receiver has been measured at approximately $5000 \mathrm{HZ}$. The bandwidth is probably less, when the effects of the receiver transducer in the telephone handset are considered. However, the fidelity of the signal is certainly greater than, or equal to, that of a normal telephone conversation. 